\title{
A High Performance Information System for College Graduation Management Cloud
}

\author{
Liumei Zhang ${ }^{1, \mathrm{a}}$, *Zhongyuan $\mathrm{Hu}^{1, \mathrm{~b}}$ \\ ${ }^{1}$ School of Computer Science, Xi'an Shiyou University, Xi'an 710065, China \\ azhangliumei@xsyu.edu.cn, ${ }^{\mathrm{b} x x @ h z y . p w}$
}

Keywords: Cloud computing, information system, application design, college graduation management.

\begin{abstract}
Traditional methods of graduation management of college students were facing challenges as the graduation number of students are increasing annually. Those methods often error pone, and the legacy systems cannot meet the requirement of fast response, broad throughput and secure of a modern application service. Therefore, in this paper a Meteor framework based high performance cloud information system is designed then implemented for college graduation management. Experiment results show that the proposed system, compared with a php implemented traditional information system, advances in response speed and throughput size. Thus, it provides a reliable platform for replacement of traditional systems of college graduation management.
\end{abstract}

\section{Introduction}

According to recent statistics, the graduation students are increasing in huge numbers annually in today's china, as can be seen from Fig. 1. There are millions of students have graduated from any type of higher education organizations. Managing such number of students traditionally require dense manual work from 7th to 8th semester in most universities. Manual process of graduation administration often error prone and time consuming. To overcome the weakness of manual administration in college graduation, there are number of higher education institutions already implemented management information systems (MIS). Those systems, compared with manual administration, has gained fame of speedy, time saving and ease of use. However, the increasing number of students has already challenged the legacy graduation MIS that fast repose speed, board throughput and better user experience are needed. Most of the legacy systems were designed and implemented with outdated architecture and pattern. They are immune to any requirements that coming from the above description, unable to be scaled up and upgraded. Therefore, in this article, a novel architecture of educational administration system for collage graduation management is proposed. The system aims at construct a platform for instantly, conveniently and securely providing student graduation services.

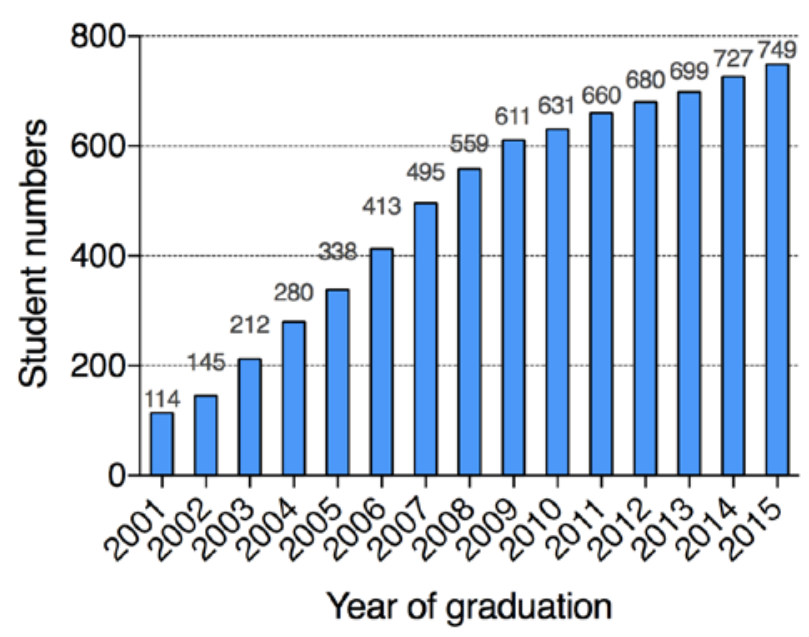

Fig. 1. Annual increasing number of college graduates 


\section{Related works}

Brumbulli et. al. presents a complex management information system designed for Albanian high schools. The application offered a suitable interface to its users in order to simplify and reduce the time needed for information and procedure management relating to different school activities ${ }^{[1]}$. With the deepening of the educational administration system and the progress of information technology, the management of education shows the tendency of disconnection between the system management and the teaching management. The author believes that the root cause lies in the lack of the overall construction of educational management system ${ }^{[2]}$. As an example of SIMS (student information management system) developed independently by School of Information Science \& Engineering of Shandong Normal University, paper ${ }^{[3]}$ introduces database design, specific realization of each function module and key technologies used in the system. Aimed at designing the software to manage the students' academic data, Norasiah et. al. designed a information system ${ }^{[4]}$. Student Record Management System (SRMS) gives a straightforward interface to support of student data. It might be utilized by instructive universities or colleges to keep up the records of students effectively ${ }^{[5]}$. The overall structure of the educational administration system, functional requirements and safety and other aspects of the design were analyzed in paper ${ }^{[6]}$. Chang et. al. applies the database into the educational management information system and gives a static model of the system through analysis of the relationship between the system class and class ${ }^{[7]}$. It uses sequence diagrams and state diagrams to in detail analyze the system's main cases, and then give the system dynamic model. Lu et. al. has introduced a kind of design process on the basis of teaching administrative system facing target's modeling ${ }^{[8]}$.

Even though the above research shows some strength on partial benefit of traditional student graduation or administration management, they are still insufficient to the modern requirement of such business logic. Therefore, a more reliable high performance information system is needed.

\section{Requirement of the System}

In most colleges of China, the design title of any student can not be same at the same year, according to the regulations. Therefore, the college will prepare the number of design project titles for students to choose. Such numbers often exceed the number of students. This also challenges the college to manage such huge number of design titles. And the whole process includes preparing and choosing design titles, require a convenient information system to maintain.

Therefore, the management system must have three basic functions: preparing titles by teachers, choosing titles by students, and then auditing students' application by teachers. Each specialty has one or more dean; their duty is auditing titles in their specialty before students start to choose. The titles didn't pass the audition will be roll back to the teacher for modifying, the rest of which will be submitted to the faculty administrator. Administrator will publish them to the website, ready for choose by students in the next stage.

The application environment has the characteristics such as large number of users, dense access time, strong requirement of real-time processing, which requires the server has the strong ability of concurrency processing.

\section{Abstract Functions}

User Roles. In this system, users are divided into four roles: students, teachers, deans and administrators. Students have the simplest role, participating in the stage of choosing project title. The functions of teachers are preparing a number of project titles, and verifying the students' choice of such titles. The responsibilities of deans in the system are examine the entire faculty project titles submitted by teachers before the choosing stage, therefore has the right to revise or negate the title in auditing.Administrators have the most complex roles. They are: be able to manage all teachers, students and deans. Due to the job features in education, they need to import and export the results of choosing, and need a dashboard to display the overview of system running state. Finally, the 
announcements function is also needed to send important information to rest of system users by administrator.

Processes. The system is closed in normal times. In annual graduation seasons, the system is manually opened according to the open time provided by administrator, then teachers begin to prepare titles. The titles are submitted to the administrator after audited by deans, the administrator will audit it again and publish them for choose by students. The choosing stage for students is divided into three round, after the completion of the stage, that is, the whole process is finished.

Fig. 2 is the state transition diagram of title object in the system design. As can be seen from this chart, the process of graduation can be understood approximately.

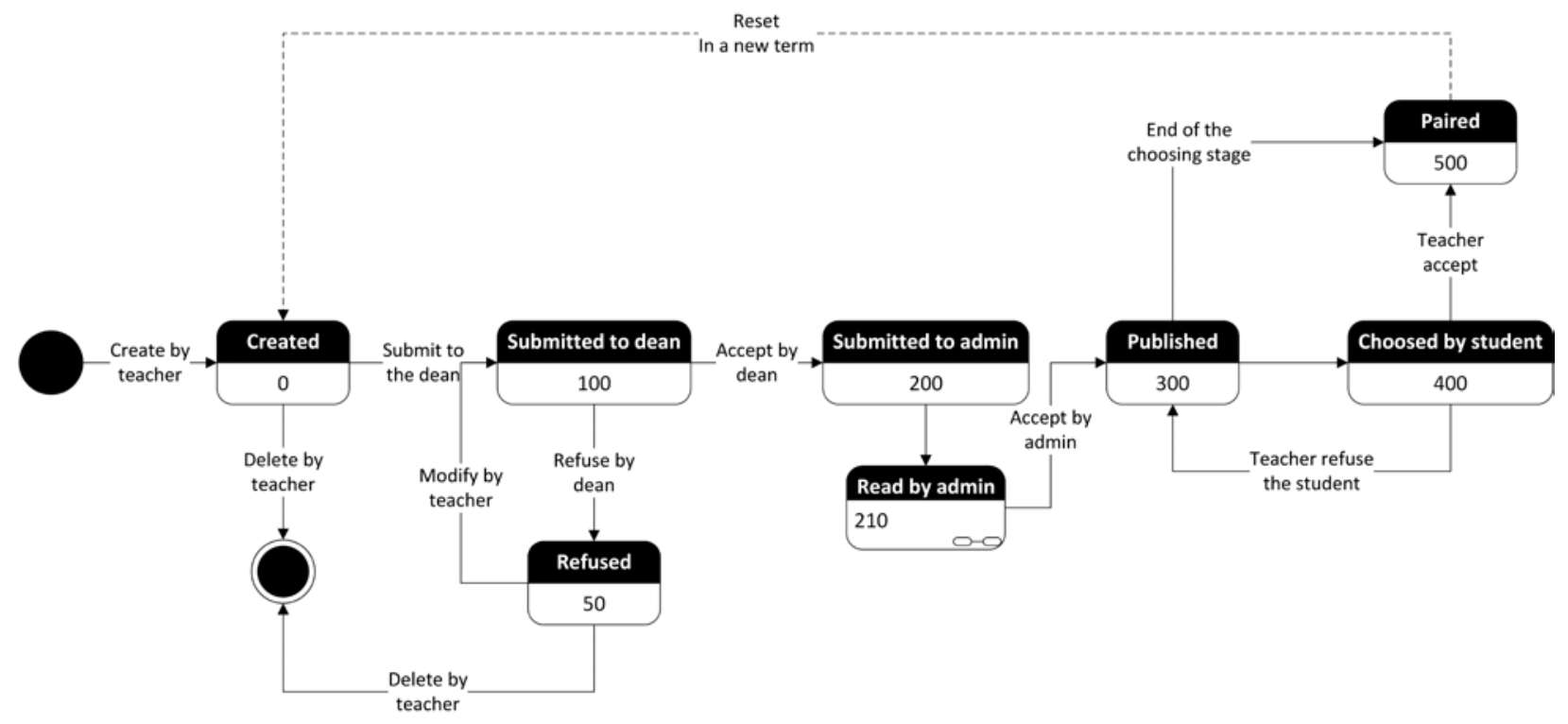

Fig. 2. The state transition diagram of title object

\section{Implementation and test}

Implementation. In this paper, we using React, MongoDB and Meteor framework to implement the system design. The system is developed based on Meteor framework, which is a modern web development tool. The traditional application server can only serve as a middle layer of data exchange between clients and the database. Meteor enables the client to operate database directly and focuses on the service logic rather than those work repeated and time-consuming. MUP (Meteor-up by Kadirahq), a third-party tool is used to deploy our Meteor app to the production server. With this tool, our system app is converted into a common Node.js app. And upload it to the configured production server, runs with Docker so gives us better security and isolation.

Load Test. Before publishing to the production environment, we use MeteorDown-v2.6.0 to running stress tests for our educational system. First, 150 title data added into title collection in MongoDB. Each title document includes 14 fields. In each test, MeteorDown will not only request to the server, also subscribe the 150 documents from title by the Meteor core method. The amount of data requested is very large, exceeding the normal use. It's enough to reflect the system stability in a high load.

The hardware configurations on the testing environment are listed as Table. 1 . And the test results are displayed as Fig. 3. 
Table. 1. The hardware configurations on the testing environment

\begin{tabular}{ll}
\hline Hardware & Details \\
\hline CPU & Intel(R) Core(TM) \\
& i7-4700MQ CPU @ \\
& 2.40GHz \\
\hline RAM & 4Gb*2 DDR3 1600MHz \\
\hline SSD & PLEXTOR PX-128M6M \\
& SATA3 \\
\hline OS & Windows 10 Professional \\
& Edition 64-bit \\
\hline
\end{tabular}

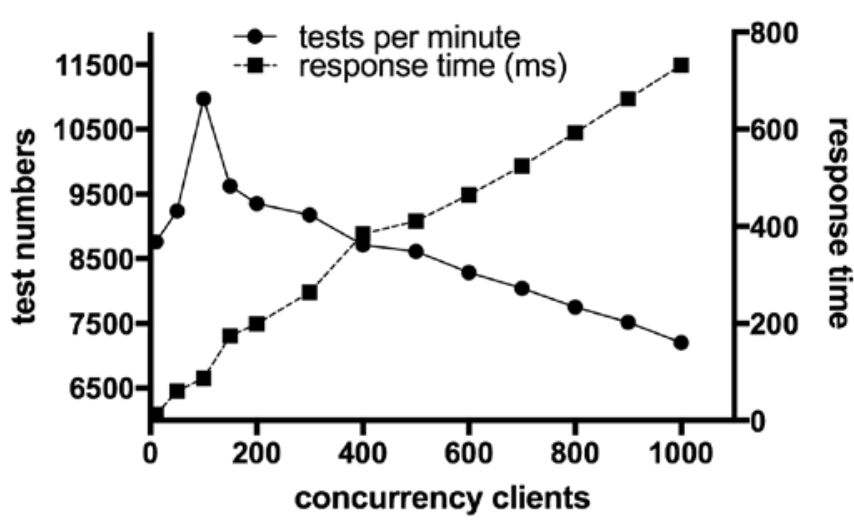

Fig. 3. The load test results

Through the test results we can see that, with the increase of the concurrent clients, although the response time is increasing gradually, but always has a satisfactory response rate. Each test needs to connect with the server side, request and download the 150 complex documents in database. Such a test cloud be executed by Meteor for nearly ten thousand times per minute, and with the increase of clients, the performance does not appear to be decreased significantly. The performance is sufficient to deploy in the production environment, for a large number of campus users at the same. The load test performance much better than the traditional models which render the HTML page by rear-end, like PHP or ASP.NET. This shows that servers only transfer data, and rendering the data by clients, the new model can reduce the server load effectively.

\section{Conclusion}

In thins paper, a high performance information system is designed and implemented for college graduation management. Such system is designed by using modern architecture design methods, then implemented based on Meteor framework. Through experiment testing, the system is verified to be stable and reliable for offering services to response high load requests.

\section{Acknowledgements}

This work was financially supported by the Xi'an Shiyou University Students Projects.

\section{References}

[1] Brumbulli, Mihal, Blerina Topçiu, and Arbora Dalaçi. SMIS: A Web-Based school management information system. International Scientific Conference Computer Science, 2008.

[2] Gao Xinjie, Modern Educational Technology 20.08(2010):20-23(in Chinese).

[3] Tang, Yu-Fang, and Yong-Sheng Zhang. Design and implementation of college student information management system based on Web Services. IT in Medicine \& Education, 2009. ITIME'09. IEEE International Symposium on. Vol. 1. IEEE, 2009.

[4] Norasiah, M. A., and A. Norhayati. Intelligent student information system. Telecommunication Technology, 2003. NCTT 2003 Proceedings. 4th National Conference on. IEEE, 2003.

[5] Walia, Er Saurabh, and Er SatinderjitKaur Gill. International Journal of Computer Science and Mobile Computing 3.8 (2014): 24-33.

[6] Mei Ya, Science \& Technology Information 31(2008):66-66.

[7] Chang, Haifeng. SQL-based educational management system design. Computer Engineering and Technology (ICCET), 2010 2nd International Conference on IEEE, 2010:V3-441 - V3-445.

[8] Lu, Yu Yang, et al. Applied Mechanics \& Materials 411-414(2013):444-447. 